\title{
The interaction of atoms with $\operatorname{LiF}(001)$ revisited
}

\author{
J.E. Miraglia ${ }^{1,2}$ and M. S. Gravielle ${ }^{1}$ \\ ${ }^{1}$ Instituto de Astronomía y Física del Espacio (IAFE, CONICET-UBA), \\ casilla de correo 67, sucursal 28, C1428EGA, Buenos Aires, Argentina. \\ ${ }^{2}$ Depto. de Física, Fac. de Ciencias Exactas y Naturales, Universidad de Buenos Aires, Argentina.
}

(Dated: September 7, 2016)

\begin{abstract}
Pairwise additive potentials for multielectronic atoms interacting with a $\mathrm{LiF}(001)$ surface are revisited by including an improved description of the electron density associated with the different lattice sites, as well as non-local electron density contributions. Within this model, the electron distribution around each ionic site of the crystal is described by means of an onion approach that accounts for the influence of the Madelung potential. From such densities, binary interatomic potentials are then derived by using well-known non-local functionals for the kinetic, exchange and correlation terms. Rumpling and long-range contributions due to projectile polarization and van der Waals forces are also included in an analogous fashion. We apply this pairwise additive approximation to evaluate the interaction potential between closed-shell - He, Ne, Ar, Kr, and Xe - and open-shell - N, S, and Cl - atoms and the LiF surface, analyzing the relative importance of the different contributions. The performance of the proposed potentials is assessed by contrasting angular positions of rainbow and supernumerary rainbow maxima produced by fast grazing incidence with available experimental data. The good agreement found for normal energies in the eV-range represents a meaningful evidence of the quality of the present description.
\end{abstract}

PACS numbers: 34.35.+a,79.20.Rf, 34.20.-b

\section{INTRODUCTION}

In the field of particle-surface interactions, one of the most remarkable experimental advances of the last decade corresponds to the observation of grazing incidence fast atom diffraction (GIFAD or FAD) [1, 2, which has emerged as a powerful surface analysis technique [36. It allows one to inspect the electronic and morphological characteristics of crystal surfaces with an exceptional sensitivity, becoming a useful tool for investigating a wide variety of materials $[7-10$.

The accuracy of the surface information provided by the FAD method crucially relies on the theoretical model used to describe the surface potential. In previous articles [11 13] we investigated the FAD process for different atoms impinging on $\operatorname{LiF}(001)$ by using a pairwise additive approach to represent the surface interaction. Pairwise additive potentials are built as a sum of binary interatomic potentials that describe the interaction of the atomic projectile with individual ionic centers of the crystal. For insulator materials, like $\mathrm{LiF}$, where the electron density is highly localized around the atomic nuclei, this simple potential model has shown to represent a reliable alternative to more complex self-consistent ab-initio calculations 12 16. However, in most works the binary potentials are derived by using the Local Density Approximation (LDA) to evaluate the kinetic and exchange terms [17. But the LDA does not include contributions due to non-local electron density terms, which might play an important role, especially for open-shell projectiles, with partially occupied outer shells.

In Ref. [18] an attempt to include the proper asymptotic limit of the binary interatomic potentials was done for the case of multielectronic atoms grazingly scattered off a $\operatorname{LiF}(001)$ surface. In this article we revisit such pairwise additive model by incorporating non-local contributions of the electron density, together with the improvement of the description of the electron density associated with each ionic center of the insulator.

The interaction between rare gases (closed-shell atoms) - He, Ne, Ar, Kr, and Xe - with fully occupied valence shells, as well as open-shell atoms - N, S, and $\mathrm{Cl}$ - with vacancies in the outer level, and a $\operatorname{LiF}(001)$ surface is studied. In all the cases, the kinetic, exchange and correlation terms of the binary potentials are evaluated from well established non-local functionals, which depend on first- and second- order derivatives of the electron density. The electron density corresponding to each ionic center of the LiF crystal is obtained from an onion model that takes into account the influence of the whole crystal lattice, i.e., the Madelung potential [19]. Long-range contributions associated with polarization and van der Waals (vdW) forces, produced by the rearrangement of the atom and surface densities as a result of the mutual interaction, are determined within a similar pairwise additive scheme. Furthermore, a surface rumpling with a displacement distance extracted from ab-initio calculations [20] is considered.

With the aim of testing the proposed potential model, we use it to evaluate angular distributions of fast atoms grazingly scattered from the LiF surface along lowindexed crystallographic channels. The elastic collision process is described within the surface initial-value representation (SIVR) approximation [21 23], which is a semiquantum method that offers a very good representation of the diffraction spectra, without requiring the use of convolutions to smooth the theoretical curves [24]. The validity of the surface potential model is examined by 
comparing the angular positions of rainbow and supernumerary rainbow maxima with available experimental data. The rainbow peak corresponds to the outermost maximum of the projectile distribution, which has a classical origin, while supernumerary rainbows are associated with FAD, being produced by quantum interference inside the channel. These deflection angles are extremely sensitive to the corrugation of the surface potential across the incidence direction, resulting a useful magnitude to probe surface interactions.

The article is organized as follows. The constituent parts of the binary interatomic potentials are summarized in Sec. II. In this section we also show the shortrange binary potentials for the different atomic projectiles - He, N, Ne, S, Ar, Cl, Kr and Xe - interacting with $\mathrm{LiF}(001)$, examining their asymptotic limits. In Sec. III we derive the corresponding total atom-surface potentials, including projectile polarization and vdW contributions. In Sec. IV, angular positions of rainbow and supernumerary rainbow maxima produced by projectile incidence along the $\langle 110\rangle$ and $\langle 100\rangle$ channels of the LiF crystal are compared with experimental data in order to illustrate the soundness of the potential model. In such a comparison, normal energies, associated with the projectile motion perpendicular to the axial channel, in the range from 0.2 to $80 \mathrm{eV}$ are considered. In Sec. V we outline our conclusions. Atomic units $\left(e^{2}=\hbar=m_{e}=1\right)$ are used unless otherwise stated.

\section{BINARY INTERACTION MODEL}

Within a pairwise additive scheme, the interaction between an impinging atom and an ionic crystal surface, like $\mathrm{LiF}(001)$, is described as a sum of binary interatomic potentials which depend on the electron densities corresponding to the atom and individual ionic centers of the crystal. In this section we will summarize all steps required to build our binary interatomic potentials, analyzing separately the asymptotic limits of each contribution.

\section{A. Ionic centers of the crystal: Onions}

With the purpose of determining the electron density corresponding to each ionic center of the LiF crystal, let us consider a perfect cubic piece of crystal centered on an active fluor ion. For this $\mathrm{F}^{-}$anion, immersed in the $\mathrm{LiF}$ crystal, the corresponding multielectronic wave function $\Psi_{\mathrm{F}}$ can be obtained by solving the Schrödinger equation associated with the Hamiltonian

$$
H_{\mathrm{F}}=\sum_{l=1}^{10}\left(-\frac{1}{2} \nabla_{\mathbf{r}_{l}}^{2}-\frac{Z_{\mathrm{F}}}{r_{l}}+V_{G}^{+}\left(\mathbf{r}_{l}\right)\right)+\frac{1}{2} \sum_{\substack{k, l=1 \\ k \neq l}}^{10} \frac{1}{r_{k l}}
$$

where $\mathbf{r}_{l}$ is the position vector of the $l$ - electron $(l=$ $1, \ldots, 10)$ with respect to the $\mathrm{F}^{-}$nuclear charge $Z_{\mathrm{F}}=9$, $r_{k l}=\left|\mathbf{r}_{k}-\mathbf{r}_{l}\right|$ is the interelectronic distance, and $V_{G}^{+}$is the potential created by the whole crystal grid, excluding the active $\mathrm{F}^{-}$ion. In Eqs. (6) and (11) of Ref. [19] $V_{G}^{+}$ was approximated by a radial onion potential produced by a series of charged shells. But these discrete charged layers introduce structures in the potential, which are difficult to handle. Therefore, in this work we fit the previous grid potential [19] by means of a smooth potential, here named Madelung potential, defined as

$$
\begin{aligned}
V_{G}^{+}(\mathbf{r}) & \simeq V_{M}^{+}(r)=-\frac{1}{r}+\frac{e^{-r / \lambda}}{r}\left[1+\left(1-\lambda V_{M 0}\right) \frac{r}{\lambda}\right. \\
& \left.+\left(\frac{1}{2}-\lambda V_{M 0}\right)\left(\frac{r}{\lambda}\right)^{2}\right]
\end{aligned}
$$

where $V_{M 0}=0.4600$ a.u. represents the proper Madelung potential at the origin [25] and the parameter $\lambda$ is chosen to verify that the spacial integral of $V_{M}^{+}(r)$ in the range $(0,+\infty)$ coincides with the one of the grid potential of Ref. [19], finding $\lambda \simeq 0.3 a / 2$, with $a=7.60$ a.u. being the lattice constant. In Fig. 1 the potential $V_{M}^{+}(r)$ is plotted along with the grid potential of Ref. [19]. Notice that $V_{M}^{+}(r)$ yields the correct asymptotic limit at long distances, i.e., $V_{M}^{+}(r) \rightarrow-1 / r$ as $r \rightarrow+\infty$, reproducing the Coulomb potential originated by an unitary charge which renders the Coulomb hole that the electron leaves behind when it is removed.

In a similar way, the two-electron wave function $\Psi_{\mathrm{Li}}$ corresponding to an active $\mathrm{Li}^{+}$cation, immersed in the $\mathrm{LiF}$ crystal, can be derived within an onion treatment from the approximate Hamiltonian

$$
H_{\mathrm{Li}} \cong \sum_{l=1}^{2}\left(-\frac{1}{2} \nabla_{\mathbf{r}_{l}}^{2}-\frac{Z_{\mathrm{Li}}}{r_{l}}+V_{M}^{-}\left(r_{l}\right)\right)+\frac{1}{2} \sum_{\substack{k, l=1 \\ k \neq l}}^{2} \frac{1}{r_{k l}},
$$

where $Z_{\mathrm{Li}}=3$ is the $\mathrm{Li}^{+}$nuclear charge and $V_{M}^{-}(r)=$ $-V_{M}^{+}(r)$.

For convenience, we call onions to these dressed anion and cation, denoting them as $F_{@}^{-}$and $L i_{@}^{+}$, respectively. The unperturbed electron density associated with each individual onion - $F_{@}^{-}$or $L i_{@}^{+}$- is obtained from the square modulus of the corresponding wave function, $\Psi_{\mathrm{F}}$ or $\Psi_{\mathrm{Li}}$, respectively. To evaluate the electronic wave functions $\Psi_{\mathrm{F}}$ and $\Psi_{\mathrm{Li}}$ we carried out full Hartree-Fock (HF) calculations from the Hamiltonians of Eqs. (1) (with $V_{M}^{+}$instead of $V_{G}^{+}$) and (3), respectively, using the code NRHF by Johnson [26. The original code was adapted to incorporate the central potential $V_{M}^{ \pm}(r)$ and a grid of about $10^{3}$ points was used in the numerical calculation.

Binding energies and mean radii derived from the electronic wave functions $\Psi_{\mathrm{F}}$ and $\Psi_{\mathrm{Li}}$ are tabulated in Table I. From this table, the binding energy of the $F_{@}^{-}(2 p)$ is about $-15 \mathrm{eV}$ ( -0.553 a.u.), agreeing fairly well with the experimental finding of $(-13 \pm 0.3) \mathrm{eV}$ for the center of the surface valence band, measured with respect 
to the vacuum level [27]. Also the ionization energies of $F_{@}^{-}(2 s)$ and $L i_{@}^{+}(1 s)$ are near to the experimental values [28], while the ionization energy of the inner state $F_{@}^{-}(1 s)$ is very close to the value obtained as the energy of the isolated $\mathrm{F}^{-}(1 s)$ [29] minus $V_{M 0}$.

\section{B. Binary interatomic potentials}

Making use of the electron densities derived within the onion model (Sec. II A), in this subsection we calculate the binary interatomic potential between an onion $O$ of the crystal surface, with $O=F_{@}^{-}$or $O=L i_{@}^{+}$, and the impinging atom $A$, as a function of the internuclear separation $R$. This binary potential is here split into two terms: one named short-range that describes the static interaction between the atomic projectile and the ionic center $O$ by considering their respective electron distributions as frozen, and the other, called long-range, which takes into account the rearrangement of their electron densities as a result of the mutual interaction, but in a perturbative way.

\section{The short-range binary potential}

The short-range potential that represents the static interaction of a neutral atom $A$, of nuclear charge $Z_{A}$ and electron density $n_{A}=n_{A}(\mathbf{r})$ [29], with an onion $O$, of nuclear charge $Z_{O} \quad\left(Z_{F_{@}^{-}}=9\right.$ and $\left.Z_{L i_{@}^{+}}=3\right)$ and electron density $n_{O}=n_{O}(\mathbf{r})$, can be approximated as a sum of four terms [30, 31]:

$$
V_{A O}^{(s h o r t)}(R)=V_{e}(R)+V_{k}(R)+V_{x}(R)+V_{c}(R),
$$

where $\mathbf{R}$ is the internuclear vector and $V_{e}, V_{k}, V_{x}$, and $V_{c}$ are the electrostatic, kinetic, exchange and correlation potentials, respectively. Due to the spherical symmetry of the interacting partners, these partial potentials depend only on $R=|\mathbf{R}|$.

As explained in Sec. II A, the electron densities $n_{A}$ and $n_{O}$ are here obtained from full HF calculations. Then, our task is to use them to build the partial potentials involved in Eq. (4). The first term of Eq. (4) represents the well-known electrostatic interaction, which reads

$$
\begin{aligned}
V_{e}(R) & =\frac{Z_{A} Z_{O}}{R}-\int d \mathbf{r}^{\prime} \frac{Z_{A} n_{O}(\mathbf{r})}{|\mathbf{r}-\mathbf{R}|}-\int d \mathbf{r} \frac{Z_{O} n_{A}\left(\mathbf{r}^{\prime}\right)}{\left|\mathbf{r}^{\prime}+\mathbf{R}\right|} \\
& +\iint d \mathbf{r} d \mathbf{r}^{\prime} \frac{n_{A}\left(\mathbf{r}^{\prime}\right) n_{O}(\mathbf{r})}{\left|\mathbf{R}+\mathbf{r}^{\prime}-\mathbf{r}\right|}
\end{aligned}
$$

while the remaining terms $-V_{k}, V_{x}$, and $V_{c}$ - can be derived as 31:

$V_{j}(R)=E_{j}\left[n_{t o t}(\mathbf{R})\right]-E_{j}\left[n_{O}\right]-E_{j}\left[n_{A}\right], \quad$ for $j=k, x, c$

by assuming that the total electron density of the atomonion system at a given internuclear separation $\mathbf{R}$ is given by [31]

$$
n_{t o t}(\mathbf{R})=n_{O}(\mathbf{r})+n_{A}(\mathbf{r}-\mathbf{R}) .
$$

In Eq. (6), the functionals $E_{j}[n]$ for $j=k, x, c$ represent the kinetic, exchange and correlation energies, respectively, depending not only on the local electron density $n(\mathbf{r})$, but also on non-local magnitudes, like the gradient and the Laplacian of the electron density.

In previous articles [12, 13] we used the spin-restricted LDA to evaluate $E_{k}$ and $E_{x}$ ( $E_{c}$ was neglected). In this article we do a quality leap by introducing non-local approximations in terms of $\nabla n$ and $\nabla^{2} n$, which allows us to obtain more accurate values, but without losing the simplicity of Eq. (6). For the exchange energy, $j=x$, we use the well established spin-dependent Becke (B) approximation given by Eq. (8) of Ref. [32]:

$$
E_{x}^{(B)}[n]=c_{x} \int d \mathbf{r} n(\mathbf{r})^{4 / 3}(1+\beta G(\mathbf{r})),
$$

where

$$
G(\mathbf{r})=\frac{g(\mathbf{r})^{2}}{1+\gamma g(\mathbf{r}) \sinh ^{-1}[g(\mathbf{r})]}
$$

with

$$
g(\mathbf{r})=\left|\nabla_{\mathbf{r}} n(\mathbf{r})\right| / n(\mathbf{r})^{4 / 3},
$$

and $c_{x}, \beta$ and $\gamma$ are constants [32]. Accordingly, for the kinetic term, $j=k$, we use the Lee-Lee-Parr (LLP) approach given by Eq. (7) of Ref. [33], which can be considered in a level equivalent to the $\mathrm{B}$ exchange expression since in terms of the Density Functional Theory it is called "conjointness":

$$
E_{k}^{(L L P)}[n]=c_{k} \int d \mathbf{r} n(\mathbf{r})^{5 / 3}(1+\alpha G(\mathbf{r})),
$$

where $c_{k}$ and $\alpha$ are constants 33 .

For the correlation energy, $j=c$, we use the celebrated Lee-Yang-Parr (LYP) approximation, given by Eqs. (21) and (22) of Ref. [34], which are valid for closed- and open- shell atoms, respectively, also including $\nabla^{2} n$. Hence, our full approximation for kinetic, exchange and correlation terms should be called with the long acronym LLPB3LYP that means Lee-LeeParr+Becke+3-coefficient-Lee-Yang-Parr [35].

For the two different onions - $F_{@}^{-}$or $L i_{@}^{+}-$in Table II we display : $(i)$ the total energy calculated from HF $\left(E_{t o t}^{(H F)}\right) ;(i i)$ the kinetic energy calculated from HF $\left(E_{k}^{(H F)}\right)$, compared with values derived from the LDA 31] $\left(E_{k}^{(L D A)}\right)$ and from the LLP approximation as given by Eq. $1133\left(E_{k}^{(L L P)}\right)$; (iii) the exchange energy calculated from $\mathrm{HF}\left(E_{x}^{(H F)}\right)$, compared with values derived from the LDA 31. $\left(E_{x}^{(L D A)}\right)$ and from the B approach as given by Eq. $832\left(E_{x}^{(B)}\right)$; and $(i v)$ the correlation 
energy evaluated by using the LYP model 34] $\left(E_{c}^{(L Y P)}\right)$. For both onions, the kinetic and exchange energies derived with the functionals of Eqs. (11) and (8), respectively, are in better agreement with the corresponding HF values than the ones obtained from the LDA. Also the total energies obtained including the correlation term $E_{c}^{(L Y P)}$ are close to the total HF values. In this regard, it is important to remind that the Virial theorem does not hold for this case because we are not dealing with a central Coulomb potential.

Results of our LLPB3LYP approximation for the short-range binary potentials corresponding to $F_{@}^{-}$and $L i_{@}^{+}$interacting with closed-shell atoms - He, Ne, Ar, Kr, Xe- are displayed in Fig. 2. In turn, in Fig. 3 we focus on projectiles having open outer shells - $\mathrm{N}\left({ }^{4} S\right), \mathrm{S}\left({ }^{3} P\right)$ and $\mathrm{Cl}\left({ }^{2} P\right)$ - which are ferromagnetic atoms corresponding to the so-called unrestricted spin cases. For these latter projectiles, as well as for $\mathrm{He}, \mathrm{Ne}$ and $\mathrm{Kr}$, there are experimental data of rainbow and/or FAD maxima available in the literature [13, 18. In Figs. 2 and 3, in order to analyze straightforwardly the asymptotic limits of the short-range potentials, results are displayed by means of the function

$$
F_{A O}^{(\text {short })}(R)=V_{A O}^{(\text {short })}(R) R\left(1+2 R^{3}\right)
$$

which makes evident the behavior at short and long distances. At the origin $F_{A O}^{(\text {short })}(0)=Z_{A} Z_{O}$, indicating that the internuclear atom-onion interaction, given by the first term of Eq. (5), provides the main contribution to the binary potential $V_{A O}^{(\text {short })}$ for small $R$ values. At large distances, instead, $F_{A O}^{(\text {short })}(R)$ tends as $V_{A O}^{(\text {short })}(R) 2 R^{4}$, which competes directly with the polarizability of the impinging atom $\alpha_{A}$ (see Eqs. (13) and (14) below). Besides, as in these figures we are plotting the absolute value of $F_{A O}^{(s h o r t)}(R)$, its sign must be indicated: at short distances $V_{A O}^{(\text {short })}$ is always positive due to the static and kinetic contributions, while at large distances $V_{A O}^{(s h o r t)}$ is negative as a consequence of the preponderance of the exchange and correlation energies.

\section{The long-range binary potential}

By long-range binary potential we mean the potential produced by the rearrangement of the electron densities of the interacting partners, also known as dispersive force, which dominates the long-distance behavior of the atom-onion interaction. Within a perturbative treatment, the long- range binary potential for the system composed by a target onion $O$, with $O=F_{@}^{-}$or $O=$ $L i_{@}^{+}$, and an incident neutral atom $A$ can be expanded as a power series of $R$, reading [36, 37]:

$$
V_{A O}^{(\text {long })}(R) \rightarrow-\frac{C_{A O}^{(4)}}{R^{4}}-\frac{C_{A O}^{(6)}}{R^{6}}-\frac{C_{A O}^{(8)}}{R^{8}}-\ldots
$$

where $R$ is again the internuclear distance. The coefficient of first term of Eq. (13) is expressed as

$$
C_{A O}^{(4)}=\frac{\alpha_{A}}{2}
$$

where $\alpha_{A}$ is the static polarizability of the atom $A$. This term is associated with the dipole momentum induced on the projectile by the target onion $O$, reflecting the contribution of the projectile polarization. In Table III we list the values of the static polarizabilities for the considered projectiles, as extracted from the bibliography [38, 39]. Furthermore, to compare the contribution of this term with the asymptotic limit of $V_{O}^{(\text {short })}$, the $\alpha_{A}$ values are also displayed in Figs. 2 and 3 considering the range $R=8-10$ a.u. where the dipolar term results relevant.

It is also interesting to investigate the following term of the expansion of Eq. (13), which is governed by the coefficient $C_{A O}^{(6)}$ related to vdW forces. The value of $C_{A O}^{(6)}$ can be estimated by using the formula of Slater-Kirkwood 40] as

$$
C_{A O}^{(6)}=\frac{3}{2} \frac{\alpha_{A} \alpha_{O}}{\left(\sqrt{\alpha_{A} / N_{A}}+\sqrt{\alpha_{O} / N_{O}}\right)},
$$

where $\alpha_{A}$ and $\alpha_{O}$ are the static polarizabilities of the atom and the onion, respectively, and $N_{A}$ and $N_{O}$ are the numbers of the corresponding active electrons (i.e., the external ones). Both magnitudes are well known for atomic projectiles: The atomic polarizabilities $\alpha_{A}$ are given in Table III, while the $N_{A}$ values can be calculated from the homonuclear coefficients $C_{A A}^{(6)}$ 39] in the usual way (see Eq. (2) of Ref. [41]). But for onions the values of $\alpha_{O}$ and $N_{O}$ must be specifically determined as explained in the Appendix A.

Using the onion values given by Eqs. A1 and A2, together with the recommended atomic parameters [38, 39], listed in Table III, we obtain the $C_{A O}^{(6)}$ values also tabulated in the same table for the different atomonion systems. For He atoms, our $C_{A O}^{(6)}$ coefficients are very close to the ones by Celli et al. [42], obtained by fitting experiments of helium atoms bound to a LiF surface. Moreover, from the $C_{A O}^{(6)}$ values of Table III we can evaluate the vdW contribution to the function

$$
F_{A O}^{(l o n g)}(R)=V_{A O}^{(l o n g)}(R) 2 R^{4},
$$

which reads $C_{A O}^{(6)} 2 R^{-2}$, also shown in Figs. 2 and 3 for $R \geq 10$ a.u. By comparing this contribution with $F_{A O}^{(s h o r t)}$ and with the projectile polarizability, we are able to estimate that vdW forces affect binary interatomic potentials only at very long distances, larger than $10 \mathrm{a} . \mathrm{u}$.

Finally, before tackling the evaluation of the total atom-surface potential, it is interesting to use the same potential model to address the study of the inter-onion $F_{@}^{-}-F_{@}^{-}, F_{@}^{-}-L i_{@}^{+}$and $L i_{@}^{+}-L i_{@}^{+}$potentials, shown in Fig. 4 (a). From these potentials we evaluate the total 
energy per onion-pair at the bulk [43], which is displayed in Fig. 4 (b) as a function of the nearest-neighbor onion distance $s_{o}$. We can see that the curve of Fig. 4 (b) presents a minimum around $s_{o}=3.8$ a.u., which is in very good agreement with the nearest-neighbor distance corresponding to the real crystal, i.e. $s_{o}=a / 2$.

\section{TOTAL ATOM-SURFACE POTENTIAL}

By using the short- and long- range binary potentials introduced in Sec. II, we proceed to build the total atomsurface potential $W\left(\mathbf{R}_{A}\right)$ for an atom $A$ interacting with a $\operatorname{LiF}(001)$ surface. It reads:

$$
W\left(\mathbf{R}_{A}\right)=W^{(\text {short })}\left(\mathbf{R}_{A}\right)+W^{(\text {long })}\left(\mathbf{R}_{A}\right),
$$

where $\mathbf{R}_{A}$ denotes the position of $A$ with respect to origin of the frame of reference, placed on a given ionic center of the topmost atomic layer (in our case, an $F_{@}^{-}$site) and $W^{(\text {short })}\left(W^{(\text {long })}\right)$ represents the short- (long-) range contribution to the total atom-surface potential.

The term $W^{(\text {short })}\left(\mathbf{R}_{A}\right)$ is expressed as the sum of the binary short-range potentials given by Eq. (4) as:

$$
W^{(\text {short })}\left(\mathbf{R}_{A}\right)=\sum_{i} e_{i} V_{A O_{i}}^{(\text {short })}\left(\rho_{i}\right),
$$

where $\rho_{i}=\mathbf{R}_{A}-\mathbf{R}_{i}$, with $\mathbf{R}_{i}$ being the position vector of the onion labeled with index $i\left(O_{i}\right)$, and the factor $e_{i}$ describes the Evjen caging, that is, $e_{i}=1$ except for onions placed at the limiting surface $\left(e_{i}=1 / 2\right)$, at the arista $\left(e_{i}=1 / 4\right)$ or at the vertex $\left(e_{i}=1 / 8\right)$ of the crystal sample. This caging warranties the Coulomb neutrality of the considered portion of crystal. In all our calculations, the sum on $i$ includes $11 \times 11 \times 4=484$ crystal sites. In addition, we considered a surface rumpling with an outward (inward) shift of the positions of the topmost $F_{@}^{-} \quad\left(L i i_{@}^{+}\right)$onions, relative to the unreconstructed surface, of 0.046 a.u., as extracted from the $a b$-initio calculation of Ref. 20]. In Fig. 5 we plot the short-range potential $W^{(\text {short })}$ for the atoms investigated in this work, as a function of the normal distance to the surface, considering a position on top of an $F^{-}$site.

In contrast with the short-range contribution, the longrange interaction $W^{(l o n g)}\left(\mathbf{R}_{A}\right)$ cannot be obtained by simply adding the corresponding binary potentials. The total long-range potential is here split in two terms:

$$
W^{(l o n g)}\left(\mathbf{R}_{A}\right)=U^{(d i p)}\left(\mathbf{R}_{A}\right)+U^{(v d W)}\left(\mathbf{R}_{A}\right),
$$

where $U^{(d i p)}$ and $U^{(v d W)}$ correspond to the dipole and vdW contributions, which are associated with the first and second term of Eq. (13), respectively. We stress that each of these contributions is not pairwise additive.

The dipole potential $U^{(d i p)}\left(\mathbf{R}_{A}\right)$ depends on the total electric field produced by the different ionic centers of the crystal, evaluated at the position of the atom $A$. It reads

$$
U^{(d i p)}\left(\mathbf{R}_{A}\right)=-\frac{\alpha_{A}}{2}\left|\sum_{i} \mathbf{E}_{i}\left(\mathbf{R}_{A}\right)\right|^{2},
$$

where

$$
\mathbf{E}_{i}\left(\mathbf{R}_{A}\right)=f_{i}\left(\rho_{i}\right) Z_{O_{i}}^{(\infty)} \frac{\widehat{\rho}_{i}}{\rho_{i}^{2}}
$$

is the electric field produced by the asymptotic charge of the onion $O_{i}$, with $Z_{O_{i}}^{(\infty)}=-1(+1)$ for $O_{i}=F_{@}^{-}\left(L i_{@}^{+}\right)$, $\widehat{\rho}_{i}=\rho_{i} / \rho_{i}$, and $f_{i}\left(\rho_{i}\right)$ is a screening factor that avoids the divergence of this electric field at the origin. In this work the function $f_{i}$ was evaluated taking into account information about the physics of adatoms, as explained in the Appendix B. Noteworthily, the projectile polarization term given by Eq. 20) strongly affects FAD spectra for incidence along the $\langle 110\rangle$ channel [11, 13. But for incidence along the $\langle 100\rangle$ direction, the alternation of the opposite effective Coulomb charges of the $F_{@}^{-}$and $L i_{@}^{+}$ onions along the channel makes the polarization effect negligible [11, 13.

In the case of the vdW contribution $U^{(v d W)}\left(\mathbf{R}_{A}\right)$, for the sake of simplicity we evaluate it at a position on top of an $\mathrm{F}^{-}$site; that is, at $\mathbf{R}_{A}=z_{A} \widehat{\mathbf{z}}$, where the versor $\widehat{\mathbf{z}}$ is oriented normal to the surface, aiming towards the vacuum region. In the most simple model, far from the surface $U^{(v d W)}\left(z_{A} \widehat{\mathbf{z}}\right)$ can be derived as the superposition of the binary vdW contributions (second term of Eq. (13) ) produced by a continuous distribution of onions, reading

$$
\begin{aligned}
U^{(v d W)}\left(z_{A} \widehat{\mathbf{z}}\right) & \rightarrow \sum-\delta_{v} \int_{-\infty}^{0} d z_{o} \int_{-\infty}^{+\infty} d x_{o} d y_{o} \\
& \times \frac{\left(C_{A F_{\Phi}^{-}}^{(6)}+C_{A L i_{@}^{+}}^{(6)}\right)}{\left[\left(z_{A}-z_{o}\right)^{2}+x_{o}^{2}+y_{o}^{2}\right]^{3}} \\
& \simeq-\frac{D^{(v d W)}}{\left(z_{A}-\bar{d}\right)^{3}} \quad \text { for } z_{A} \rightarrow+\infty,
\end{aligned}
$$

where

$$
D^{(v d W)}=\frac{\pi}{6} \delta_{v}\left(C_{A F_{@}^{-}}^{(6)}+C_{A L i_{@}^{+}}^{(6)}\right),
$$

$\delta_{v}=4 / a^{3}$ is the volume density of each onion and $\bar{d}$ is a reference distance that does not have direct relation with the equilibrium position.

In relation to Eq. 222, we must mention that it does not take into account the fact that the different dipoles induced in the crystal by the projectile interaction screen each other 42 . For this reason, $D^{(v d W)}$ values derived from Eq. (23) should be considered as an upper limit because they are expected to be higher than those obtained from the approach by Lifshitz et al. 44, 45, which includes the proper screening. Remarkably, we observe that the $z_{A}^{-3}$ dependence given by Eq. 22, , which gave rise to the famous potential $V_{9-3}$ [45], starts to dominate at distances farther than 10 a.u. This fact makes the influence of $U^{(v d W)}$ negligible for normal energies higher 
than $0.2 \mathrm{eV}$, like the ones considered in this article, where closest distances smaller than 5.3 a.u. are reached by the impinging atoms.

Concerning the importance of the vdW contribution, we should draw the attention to $\mathrm{H}$ projectiles, for which a completely different situation is observed. In the case of $\mathrm{FAD}$ for $\mathrm{H}$ on $\mathrm{LiF}(001)$, a non-negligible role of $\mathrm{vdW}$ interactions was recently reported in the low-tointermediate normal energy regime [46]. Such a noticeable vdW effect is compatible with the relatively high $C_{A O}^{(6)}$ values for hydrogen atoms, in comparison with the corresponding short-range potentials, obtained within our onion model: $C_{H F_{@}^{-}}^{(6)}=15.1$ a.u. and $C_{H L i_{@}^{+}}^{(6)}=0.47$ a.u.

\section{COMPARISON WITH GRAZING INCIDENCE EXPERIMENTS}

With the goal of checking the quality of the proposed surface potential we use the potential model within the framework of the SIVR approximation in order to evaluate final projectile distributions for swift atoms grazingly impinging on $\mathrm{LiF}(001)$ along low-indexed crystallographic channels. The SIVR approach [21, 22] is a semiquantum method that provides a clear representation of the main physical mechanisms involved in FAD processes, describing them in terms of classical trajectories through the Feynman path integral formulation of quantum mechanics [47. It incorporates an adequate description of classically forbidden transitions on the dark side of the rainbow angle, providing reliable FAD patterns along the whole angular range 21, 22.

Under axial incidence conditions [4, like the ones considered here, the angular distribution of scattered projectiles lays on an annulus given by $\varphi_{f}^{2}+\theta_{f}^{2} \approx \theta_{i}^{2}$, where $\theta_{f}$ $\left(\theta_{i}\right)$ is the final (initial) polar angle, measured with respect to the surface, and $\varphi_{f}$ is the azimuthal exit angle measured with respect to the incidence direction in the surface plane, as shown in the inset of Fig. 6. The typical FAD distribution displayed in such an inset presents maxima symmetrically placed with respect to $\varphi_{f}=0$, which are associated with rainbow and supernumerary rainbow peaks. The outermost maxima of the spectrum are produced by rainbow scattering, having a classical explanation, while the inner peaks are related to supernumerary rainbows, being originated by quantum interference among trajectories with starting positions placed inside one reduced unit cell of the crystal surface. Furthermore, for LiF surfaces when the final projectile distribution is plotted as a function of the deflection angle $\Theta$, defined as $\Theta=\arctan \left(\varphi_{f} / \theta_{f}\right)$, both the position and intensity of the peaks become completely governed by the normal energy $E_{\perp}=E \sin ^{2} \theta_{i}$, which is related to the motion in the plane perpendicular to the incident channel, with $E$ being the impact energy. The angular positions of the peaks are strongly affected by the corrugation of the atom-surface potential across the axial direction, making possible to probe the potential model for different distances to the surface by varying the $E_{\perp}$ value. Notice that for low normal energies, both rainbow and supernumerary rainbow peaks are present in FAD spectra, but when $E_{\perp}$ increases supernumerary rainbows start to blur out, and for large energies only rainbow structures are observed in the projectile distributions.

To show an overall picture of performance of the proposed potential model, in Fig. 6 we plot the deflection angle corresponding to the rainbow maximum, $\Theta_{\mathrm{rb}}$, as a function of $E_{\perp}$, for closed- shell (Ne and $\mathrm{Kr}$ ) and openshell $(\mathrm{S}$ and $\mathrm{Cl})$ atoms grazingly colliding with $\mathrm{LiF}(001)$ along two different channels: $\langle 110\rangle$ and $\langle 100\rangle$. In the figure, $\Theta_{\mathrm{rb}}$ values obtained from the proposed pairwise potential model are compared with available experimental data 13, 18, considering a wide normal energy range that covers from 0.2 to $80 \mathrm{eV}$. Note that for high normal energies, the angular positions of rainbow maxima derived from the SIVR method agree with those obtained from classical simulations due to the classical origin of rainbow scattering. In the SIVR calculations, the atom-surface potential was evaluated as the sum of a short-range contribution, derived from the LLPB3LYP approach, and a long-range contribution accounting for projectile polarization through the dipolar term given by Eq. 20). The $\mathrm{vdW}$ contribution was not included in the simulations because it was estimated as negligible, as explained in Sec. III.

From Fig. 6 we observe that for closed-shell projectiles, our potential model yields rainbow angles in very good accord with the experimental data in the whole $E_{\perp}$-range. But for $\mathrm{S}$ and $\mathrm{Cl}$ projectiles, both open-shell atoms, the theoretical $\Theta_{\mathrm{rb}}$ values agree with the experimental ones for $E_{\perp}$ up to 60 and $30 \mathrm{eV}$, respectively, running slightly below the experiments at higher normal energies. This underestimation of rainbow deflection angles in the high normal-energy region is associated with a less corrugated potential energy surface, which affects projectiles that reach closer distances to the surface, with maximum approach distances lower than 2.0 a.u. In Fig. 6 we also investigate the influence of the correlation term, usually left out in pairwise potential models, by plotting $\Theta_{\mathrm{rb}}$ values obtained by neglecting the correlation contribution $E_{c}$, that is, by using the LLPB approximation, instead of the LLPB3LYP one, to represent the short-range binary interactions. In all the spectra we found that rainbow deflection angles derived from the LLPB approach are very close to those obtained with LLPB3LYP, indicating a weak effect of the correlation term on rainbow scattering.

Additionally, in the low energy region it is possible to make a more exhaustive analysis of the surface potential by using FAD patterns. In Fig. 7 angular positions of rainbow and supernumerary rainbow maxima, obtained from SIVR simulations for Ne (closed-shell) and N (openshell) atoms impinging on $\operatorname{LiF}(001)$ along the $\langle 110\rangle$ direction, are compared with experimental FAD data [13, 48, as a function of $E_{\perp}$. These FAD maxima represent a 
sensitive test of the corrugation of the potential across the channel. In this regard, we have chosen the $\langle 110\rangle$ channel, instead of the $\langle 100\rangle$ one, because the former presents a higher corrugation of the potential across it, producing consequently richer diffraction patterns, with a larger number of supernumeraries. Notice that the energy range where supernumerary maxima can be experimentally resolved depends on the projectile mass [13, being smaller for Ne than for $\mathrm{N}$ impact. For Ne projectiles, the SIVR approximation, using the proposed pairwise additive potential, reproduces well the experimental positions of rainbow and supernumerary rainbow maxima over the whole energy range. But the experimental data are slightly underestimated when the correlation term is neglected in the binary potentials. On the contrary, for $\mathrm{N}$ projectiles the inclusion of the correlation term gives rise to an increase of the deflection angles corresponding to rainbow and supernumerary rainbow peaks, overestimating slightly the experimental values, which are better reproduced when the LLPB model, without correlation, is used in the calculations. Similar behavior was also observed in FAD spectra for He projectiles, case investigated with the present pairwise potential model in Refs. [21, 22].

\section{CONCLUSIONS}

We have derived a pairwise additive potential for neutral atoms interacting with a $\operatorname{LiF}(001)$ surface by using state-of-the-art methods to describe binary potentials in terms of the electron density. The model incorporates not only non-local contributions of the electron density, but also the effect of the ionic crystal lattice on the electron density around individual ionic sites of the material, here named onions. For closed-shell ( $\mathrm{He}, \mathrm{Ne}, \mathrm{Ar}, \mathrm{Kr}$, and $\mathrm{Xe}$ ) and open-shell (N, S, and $\mathrm{Cl}$ ) atoms, the shortrange and long- range contributions to the corresponding atom-onion potentials were analyzed as a function of the internuclear distance, comparing the relative importance of each term.

The degree of accuracy of the proposed atom-surface potential model was illustrated by contrasting theoretical angular positions of rainbow maxima with experimental data for axial grazing scattering with normal energies from 0.2 to $80 \mathrm{eV}$. In this energy range the vdW contribution to the surface potential was estimated as negligible and consequently, it was not included in the calculations. For two different incidence directions - $\langle 110\rangle$ and $\langle 100\rangle$ - and closed-shell projectiles, the rainbow angles derived from the proposed potential were found in excellent agreement with the experiments in the whole $E_{\perp}$ - range. For open-shell atoms, instead, an analogous accord was observed up to intermediate $E_{\perp}$ values, but in the high normal energy region our theoretical results slightly underestimate the experiment. In addition, the low $E_{\perp}$ - region of the surface potential was deeply probed by comparing theoretical and experimental angular po- sitions of rainbow and supernumerary rainbow maxima, which are produced by very sensitive FAD processes. Experimental data for closed-shell $\mathrm{Ne}$ atoms as well as for open-shell $\mathrm{N}$ atoms were fairly well reproduced by our surface potential. Furthermore, in all the cases the correlation term was found to play a minor role, its effect being appreciable for low $E_{\perp}$ - values only. Therefore, we conclude that our pairwise additive potential model can be used with confidence for closed-shell atoms in the 0.2 $-80 \mathrm{eV}$ normal energy range and for open-shell atoms up to intermediate energies. It is important to point out that such a potential model presents the advantage of allowing one to extract straightforwardly information about the different interaction mechanisms.

\section{Acknowledgments}

Experimental contribution from the group of Helmut Winter (Institut für Physik, Humboldt-Universität zu Berlin) is gratefully acknowledged. The authors thank financial support from CONICET, UBA, and ANPCyT of Argentina.

\section{Appendix A: Onion polarizabilities}

The polarizabilities of the $F_{@}^{-}$and $L i_{@}^{+}$onions, namely, $\alpha_{F_{\odot}^{-}}$and $\alpha_{L i_{\Theta}^{+}}$, should slightly differ from the polarizabilities of the free ions, given by $\alpha_{\mathrm{F}^{-}}=10.6$ a.u. and $\alpha_{\mathrm{Li}^{+}}=0.188$ a.u. for $\mathrm{F}^{-}$and $\mathrm{Li}^{+}$, respectively [38, 49]. Then, we use the fact that the polarizability is proportional to the volume [50] to estimate the onion polarizabilities as

$$
\begin{aligned}
& \alpha_{F_{@}^{-}} \simeq \frac{\left\langle r^{3}\right\rangle_{F^{-}}}{\left\langle r^{3}\right\rangle_{\mathrm{F}^{-}}} \alpha_{\mathrm{F}^{-}}=8.69 \text { a.u., } \\
& \alpha_{L i_{@}^{+}} \simeq \frac{\left\langle r^{3}\right\rangle_{L_{@}^{+}}}{\left\langle r^{3}\right\rangle_{\mathrm{Li}^{+}}} \alpha_{\mathrm{Li}^{+}}=0.191 \text { a.u. }
\end{aligned}
$$

where $\left\langle r^{3}\right\rangle_{j}$ is the mean volume of the ion $j$, with $j=$ $\mathrm{F}^{-}, \mathrm{Li}^{+}, F_{@}^{-}, L i_{@}^{+}[51$.

Concerning the number of active electrons of the onion, we exploit the rule of Ref. [41] that shows that the same number of active electrons can be applied to an entire isoelectronic sequence, no matter the charge state, without affecting the accuracy of Eq. 15. Therefore, from Table III:

$$
\begin{aligned}
& N_{F_{@}^{-}}=N_{N e}=3.59, \\
& N_{L i_{@}^{+}}=N_{H e}=1.36 .
\end{aligned}
$$




\section{Appendix B: Screening function derived from adatom eigenenergies}

In Eq. 21) we have introduced a screening function $f_{i}\left(\rho_{i}\right)$, extracted from Ref. [36, which avoids the divergence of the electric field at the origin. It reads

$$
f_{i}\left(\rho_{i}\right)=1-\exp \left(-\frac{\rho_{i}}{\mu_{i}}\right)\left[\sum_{j=0}^{2} \frac{1}{j !}\left(\frac{\rho_{i}}{\mu_{i}}\right)^{j}\right],
$$

where $\mu_{i}$ is a screening parameter defined as $\mu_{i}=$ $\eta\left(\sqrt{\left\langle r^{2}\right\rangle_{O_{i}}}+\sqrt{\left\langle r^{2}\right\rangle_{A}}\right)$, with $\left\langle r^{2}\right\rangle_{O_{i}}$ and $\left\langle r^{2}\right\rangle_{A}$ being the mean square radii corresponding to the outer shell of the onion and the atom, respectively, and $\eta$ is an external factor that is here calculated from the physics of adatoms as follows.

Atom-surface potentials calculated from Eq. 17 should support adatoms in front of the LiF surface, that is, atoms weakly bound to the surface 45. In the case of physical absorption from a surface, the binding potential is characterized by the depth of the attractive well $V_{b}$, the ground-state binding energy $E_{b}$, and the equilibrium distance $d_{b}$ from the surface, so that around this position the potential behaves as a harmonic oscillator. To compare with adatom values, we introduce an averaged potential, without corrugation (planar), valid at a large distance $d$ from the surface, as

$$
W\left(\mathbf{R}_{A}\right) \simeq \frac{1}{2} W\left(\mathbf{R}_{\mathrm{F}}\right)+\frac{1}{2} W\left(\mathbf{R}_{\mathrm{Li}}\right)+W\left(\mathbf{R}_{\text {hole }}\right)
$$

where $\mathbf{R}_{A}=\mathbf{R}_{\mathrm{F}}=(0,0, d)$ is the position upon the $F_{@}^{-}$ onion, $\mathbf{R}_{\mathrm{Li}}=(0, a / 2, d)$ is the one upon $L i_{\mathfrak{Q}}^{+}$, and $\mathbf{R}_{\text {hole }}=(a / 4, a / 4, d)$ is the position upon the center of the reduced unit cell (hole).

For He projectiles, using $\eta=0.7$ and neglecting any $\mathrm{vdW}$ contribution, we found that $V_{b}=8.0 \mathrm{meV}$ and $d_{b}=2.99 \AA$, in close agreement with the reported experimental data $8.5 \mathrm{meV}$ and $2.98 \AA$, respectively [45]. The so-obtained potential $W\left(\mathbf{R}_{A}\right)$ can be fitted with a Morse potential to give a binding energy $E_{b}=5 \mathrm{meV}$ that compares quite well with the best estimate value $5.9 \mathrm{meV}$ [45. Similarly, for Ne, using $\eta=0.72$, we found $V_{b}=12.7 \mathrm{meV}$ and $E_{b}=10.9 \mathrm{meV}$, while the reported data are 13.5 and $11.7 \mathrm{meV}$, respectively [45]. For the remaining rare gases: $\mathrm{Ar}, \mathrm{Kr}$, and Xe we use $\eta=0.67,0.64$, and 0.62 , producing $V_{b}=69,93$, and $157 \mathrm{meV}$, comparable with the reported values $70 \pm 10,94.2$, and 153 meV, respectively [45. Therefore, the $\eta$ value seems to be rather universal and situates around 0.7 , in accordance with our previous findings [13, 18, 21]. In this work we used such a value for our SIVR simulations.
[1] A. Schüller, S. Wethekam, and H. Winter. Diffraction of Fast Atomic Projectiles during Grazing Scattering from a LiF(001) Surface. Phys. Rev. Lett., 98:016103, 2007.

[2] P. Rousseau, H. Khemliche, A. G. Borisov, and P. Roncin. Quantum Scattering of Fast Atoms and Molecules on Surfaces. Phys. Rev. Lett., 98:016104, 2007.

[3] H. Khemliche, P. Rousseau, P. Roncin, V. H. Etgens, and F. Finocchi. Grazing incidence fast atom diffraction: An innovative approach to surface structure analysis. Appl. Phys. Lett., 95:151901, 2009.

[4] H. Winter and A. Schüller. Fast atom diffraction during grazing scattering from surfaces. Prog. Surf. Sci., 86:169221, 2011.

[5] P. Atkinson, M. Eddrief, V. H. Etgens, H. Khemliche, M. Debiossac, A. Momeni, M. Mulier, B. Lalmi, and P. Roncin. Dynamic grazing incidence fast atom diffraction during molecular beam epitaxial growth of GaAs. Appl. Phys. Lett., 105:021602, 2014.

[6] J. Seifert and H. Winter. Quantitative structure determination using grazing scattering of fast atoms: Oxygeninduced missing-row reconstruction of $\mathrm{Mo}(112)$. Phys. Rev. B, 93:205417, 2016.

[7] A. Schüller, M. Busch, S. Wethekam, and H. Winter. Fast Atom Diffraction from Superstructures on a Fe(110) Surface. Phys. Rev. Lett., 102:017602, 2009.

[8] J. Seifert, A. Schüller, H. Winter, R. Włodarczyk, J. Sauer, and M. Sierka. Diffraction of fast atoms during grazing scattering from the surface of an ultrathin silica film on Mo(112). Phys. Rev. B, 82:035436, 2010.

[9] J. Seifert, M. Busch, E. Meyer, and H. Winter. Surface Structure of Alanine on $\mathrm{Cu}(110)$ Studied by Fast Atom Diffraction. Phys. Rev. Lett., 111:137601, 2013.

[10] A. Zugarramurdi, M. Debiossac, P. Lunca-Popa, A. J. Mayne, A. Momeni, A. G. Borisov, Z. Mu, P. Roncin, and $\mathrm{H}$. Khemliche. Determination of the geometric corrugation of graphene on $\mathrm{SiC}(0001)$ by grazing incidence fast atom diffraction. Appl. Phys. Lett., 106:101902, 2015.

[11] M. S. Gravielle and J. E. Miraglia. Influence of the polarization in grazing scattering of fast helium atoms from LiF(001) surfaces. Phys. Rev. A, 78:022901, 2008.

[12] M. S. Gravielle and J.E. Miraglia. Quantum interference in grazing scattering of swift He atoms from $\mathrm{LiF}(001)$ surfaces: Surface eikonal approximation. Nucl. Instr. Methods Phys. Res. B, 267:610-614, 2009.

[13] M. S. Gravielle, A. Schüller, H. Winter, and J.E. Miraglia. Fast atom diffraction for grazing scattering of Ne atoms from a $\mathrm{LiF}(001)$ surface. Nucl. Instr. Methods Phys. Res. B, 269:1208-1211, 2011.

[14] A. Schüller, K. Gärtner, and H. Winter. Interaction potential for fast $\mathrm{Ne}$ atoms in front of $\mathrm{LiF}(001)$ surface. Eur. Phys. Lett., 81:37007, 2008.

[15] A. Schüller, M. Busch, J. Seifert, S. Wethekam, H. Winter, and K. Gärtner. Superstructures of oxygen and sulphur on a Fe(110) surface via fast atom diffraction. Phys. Rev. B, 79:235425, 2009.

[16] U. Specht, M. Busch, J. Seifert, A. Schüller, H. Winter, 
K. Gärtner, R. Włodarczyk, M. Sierka, , and J. Sauer. Rainbow scattering under axial surface channeling from a KCl(001) surface. Phys. Rev. B, 84:125440, 2011.

[17] A. A. Abrahamson. Repulsive interaction potentials between rare-gas atoms. Heteronuclear two-center systems. Phys. Rev., 133:A990-A1004, 1964.

[18] M. S. Gravielle, J.E. Miraglia, A. Schüller, and H. Winter. Interaction potentials for multi-electron atoms in front of a $\mathrm{LiF}$ (001) surface from rainbow scattering. Nucl. Instr. Methods Phys. Res. B, 317:77-82, 2013.

[19] J. E. Miraglia and M. S. Gravielle. Production of excitons in grazing collisions of protons with LiF surfaces: An onion model. Phys. Rev. A, 84:062901, 2011.

[20] A. Schüller, H. Winter, M. S. Gravielle, J. M. Pruneda, and J. E. Miraglia. He-LiF surface interaction potential from fast atom diffraction. Phys. Rev. A, 80:062903, 2009.

[21] M. S. Gravielle and J. E. Miraglia. Semiquantum approach for fast atom diffraction: Solving the rainbow divergence. Phys. Rev. A, 90:052718, 2014.

[22] M. S. Gravielle and J. E. Miraglia. Influence of beam collimation on fast-atom diffraction studied via a semiquantum approach. Phys. Rev. A, 92:062709, 2015.

[23] M. S. Gravielle and J. E. Miraglia. Single- and doubleslit collimating effects on fast-atom diffraction spectra. Nucl. Instrum. Methods Phys. Res. B, 382:42-48, 2016.

[24] C. A. Ríos Rubiano, G. A. Bocan, M. S. Gravielle, N. Bundaleski, H. Khemliche, and P. Roncin. Ab initio potential for the $\mathrm{He}-\mathrm{Ag}(110)$ interaction investigated using grazing-incidence fast-atom diffraction. Phys. Rev. A, 87:012903, 2013.

[25] H. Tatewaki and E. Miyoshi. The surface and bulk excitons of crystalline $\mathrm{LiF} . \mathrm{Li}_{n}^{+} \mathrm{F}_{m}^{-}$cluster embedded in an ionic cage. Surf. Sci., 327:129-144, 1995.

[26] W. Johnson. Free code at www3.nd.edu/ Johnson/Class01F/nrhf.f.

[27] P. Roncin, J. Villette, J. P. Atanas, and H. Khemliche. Energy Loss of Low Energy Protons on LiF(100): Surface Excitation and $\mathrm{H}^{-}$Mediated Electron Emission. Phys. Rev. Lett., 83:864-867, 1999.

[28] S. Samarin, J. Berakdar, A. Suvorova, O. M. Artamonov, D. K. Waterhouse, J. Kirschner, and J. F. Williams. Secondary-electron emission mechanism of LiF film by (e,2e) spectroscopy. Surf. Sci., 548:187-199, 2004.

[29] E. Clementi and C. Roetti. Roothaan-Hartree-Fock Atomic Wavefunctions. At. Data and Nucl. Data Tables, 14:177-478, 1974.

[30] Y. S. Kim and R. G. Gordon. Ion-rare gas interactions on the repulsive part of the potential curves. J Chem. Phys., 60:4323-4331, 1974.

[31] R. G. Parr and W. Yang. (Oxford University Press, New York). Density-Functional Theory of Atoms and Molecules, Chapter 10, 1989.

[32] A. D. Becke. Density-functional exchange-energy approximation with correct asymptotic behavior. Phys. Rev. A, 38:3098-3100, 1988.

[33] H. Lee, C. Lee, and R. G. Parr. Conjoint gradient correction to the Hartree-Fock kinetic- and exchange-energy density functionals. Phys. Rev. A, 44:768-771, 1991.

[34] C. Lee, W. Yang, and R. G. Parr. Development of the Colle-Salvetti correlation-energy formula into a functional of the electron density. Phys. Rev. B, 37:785-789, 1988.

[35] K. Burke. Perspective on density functional theory. J.
Chem. Phys., 136:150901, 2012.

[36] K. T. Tang and J. P. Toennies. An improved simple model for the van der Waals potential based on universal damping functions for the dispersion coefficients. $J$. Chem. Phys., 80:3726-3741, 1984.

[37] E. Meyer. Strukturuntersuchungen an Oxidkristalloberflächen mittels der streifenden Streuung schneller Atome. Habilitationsschrift, Humboldt-Universität zu Berlin, Diss., 2015.

[38] J. Mitroy, M. S. Safranova, and C. W. Clark. Theory and applications of atomic and ionic polarizabilities. J. Phys. B: At. Mol. Opt. Phys., 43:202001, 2010.

[39] X. Chu and A. Dalgarno. Linear response timedependent density functional theory for van der Waals coefficients. J. Chem. Phys., 121:4083-4088, 2004.

[40] J. C. Slater and J. G. Kirkwood. The van der Waals forces in gases. Phys. Rev., 37:682-697, 1931.

[41] A. D. Koutselos and E. A. Mason. Correlation and prediction of dispersion coefficients for isoelectronic systems. J. Chem. Phys., 85:2154-2160, 1986.

[42] V. Celli, D. Eichenauer, A. Kaufhold, and J. P. Toennies. Pairwise additive semi ab-initio potential for the elastic scattering of He atoms from the $\mathrm{LiF}(001)$ crystal surface. J. Chem. Phys., 83:2504-2521, 1985.

[43] N. W. Ashcroft and N. D. Mermin. (Brooks/Cole, Cengage Learning, Belmont, USA). Solid State Physics, Chap. 20, 1976.

[44] E. M. Lifshitz. The Theory of Molecular Attractive Forces between Solids. Sov. Phys. JETP, 2:73-83, 1956.

[45] G. Vidali, G. Ihm, H-Y. Kim, and M. W. Cole. Potentials of physical adsorption. Surf. Sci. Rep., 12:133-181, 1991.

[46] G. A. Bocan, J. D. Fuhr, and M. S. Gravielle. van derWaals effects on grazing-incidence fast-atom diffraction for $\mathrm{H}$ on $\mathrm{LiF}(001)$. Phys. Rev. A, 94:022711, 2016.

[47] W. H. Miller. The Semiclassical Initial Value Representation: A Potentially Practical Way for Adding Quantum Effects to Classical Molecular Dynamics Simulations. J. Phys. Chem A, 105:2942-2955, 2001.

[48] H. Winter. Prived communication.

[49] R. D. Shannon and R. X. Fischer. Empirical electronic polarizabilities in oxides, hydroxides, oxyfluorides, and oxychlorides. Phys. Rev. B, 73:235111, 2006.

[50] F. O. Kannemann and A. D. Becke. Atomic volumes and polarizabilities in density-functional theory. J. Chem. Phys., 136:034109, 2012.

[51] From HF calculations, the mean volumens of the $F_{@}^{-}$and $L i_{@}^{+}$onions and the $\mathrm{F}^{-}$and $\mathrm{Li}^{+}$isolate ions are $\left\langle r^{3}\right\rangle_{F_{@}^{-}}=$ 2.916 a.u., $\left\langle r^{3}\right\rangle_{L i_{@}^{+}}=0.4473$ a.u., $\left\langle r^{3}\right\rangle_{\mathrm{F}^{-}}=3.557$ a.u., $\left\langle r^{3}\right\rangle_{\mathrm{Li}^{+}}=0.4394$ a.u., respectively. 


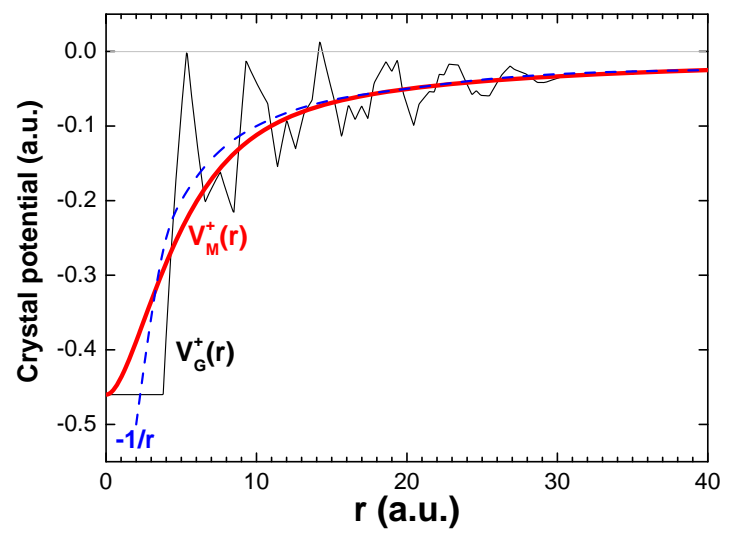

FIG. 1: (Color online) Crystal potential, as a function of the radial distance $r$ to an $\mathrm{F}^{-}$site of the crystal lattice. Red thick solid line, Madelung potential, $V_{M}^{+}$, as given by Eq. (2); black thin solid line, radial grid potential, $V_{G}^{+}$, as given by Eq. (6) of Ref. 19]; blue dashed line, asymptotic limit $-1 / r$ of the crystal potential. 


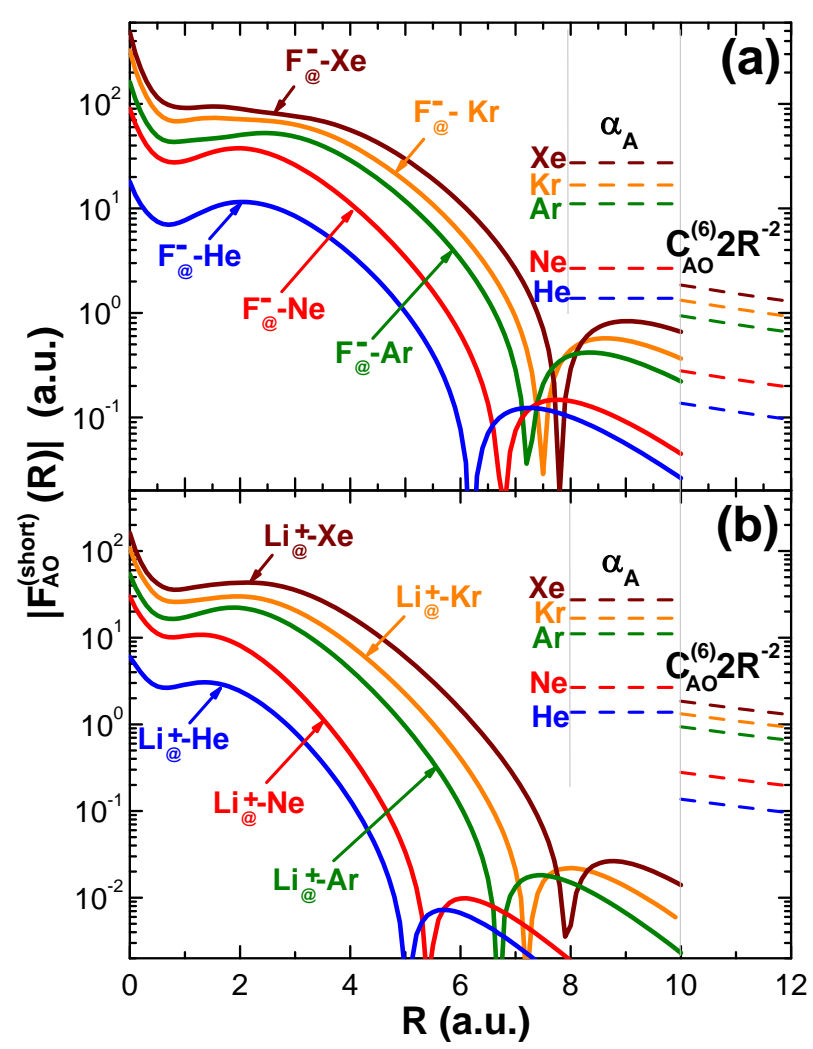

FIG. 2: (Color online) Binary interatomic potential, as a function of the internuclear distance $R$, for different closedshell atoms. (a) Absolute value of $F_{A O}^{(\text {short })}(R)$, given by Eq. (12), for the interaction of different closed-shell atoms with $F_{@}^{-}$anions. (b) Analogous to (a) for the interaction with $L i_{@}^{+}$cations. In both panels, LLPB3LYP results for different atom-onion pairs are plotted with different colors. Projectile dipole polarizabilities, $\alpha_{A}$, and van der Walls contributions, $C_{A O}^{(6)} 2 R^{-2}$, are displayed in the ranges 8 a.u. $\leq R \leq 10$ a.u. and 10 a.u. $\leq R \leq 12$ a.u., respectively, as explained in the text.

\begin{tabular}{|l|rrrrrr|rrr|}
\hline & $F_{@}^{-}(1 s)$ & $F_{\Theta}^{-}(2 s)$ & $F_{\Theta}^{-}(2 p)$ & $F_{\Theta}^{-}(3 s)$ & $F_{@}^{-}(3 p)$ & $F_{@}^{-}(3 d)$ & $L i_{\Theta}^{+}(1 s)$ & $L i_{@}^{+}(2 s)$ & $L i_{@}^{+}(2 p)$ \\
\hline$E_{n l}$ & -26.17 & -1.447 & -0.5532 & -0.1593 & -0.0994 & 0.0559 & -2.348 & -0.2249 & -0.1499 \\
$\langle r\rangle_{n l}$ & 0.1758 & 1.033 & 1.208 & 4.804 & 6.703 & 10.36 & 0.560 & 3.413 & 3.948 \\
\hline
\end{tabular}

TABLE I: HF bound energies and mean-radii for the considered onions. All the values in atomic units.

\begin{tabular}{|l|r|rrr|rrr|r|}
\hline & \multicolumn{1}{|c|}{$E_{\text {tot }}^{(H F)}$} & $E_{k}^{(H F)}$ & $E_{k}^{(L D A)}$ & $E_{k}^{(L L P)}$ & $E_{x}^{(H F)}$ & $E_{x}^{(L D A)}$ & $E_{x}^{(B)}$ & $E_{c}^{(L Y P)}$ \\
\hline$F_{@}^{-}$ & -103.766 & 99.866 & 91.151 & 99.624 & -10.545 & -9.449 & -10.423 & -0.363 \\
$L_{@}^{+}$ & -6.3414 & 7.200 & 6.507 & 7.253 & -1.656 & -1.507 & -1.640 & -0.0048 \\
\hline
\end{tabular}

TABLE II: Total $E_{t o t}$, kinetic $E_{k}$, exchange $E_{x}$ and correlation $E_{c}$ energies calculated with HF, LDA, LLP 33. (Eq. 11), B 32] (Eq. (8)), and LYP 34, respectively, for the two onions. All the values in atomic units. 


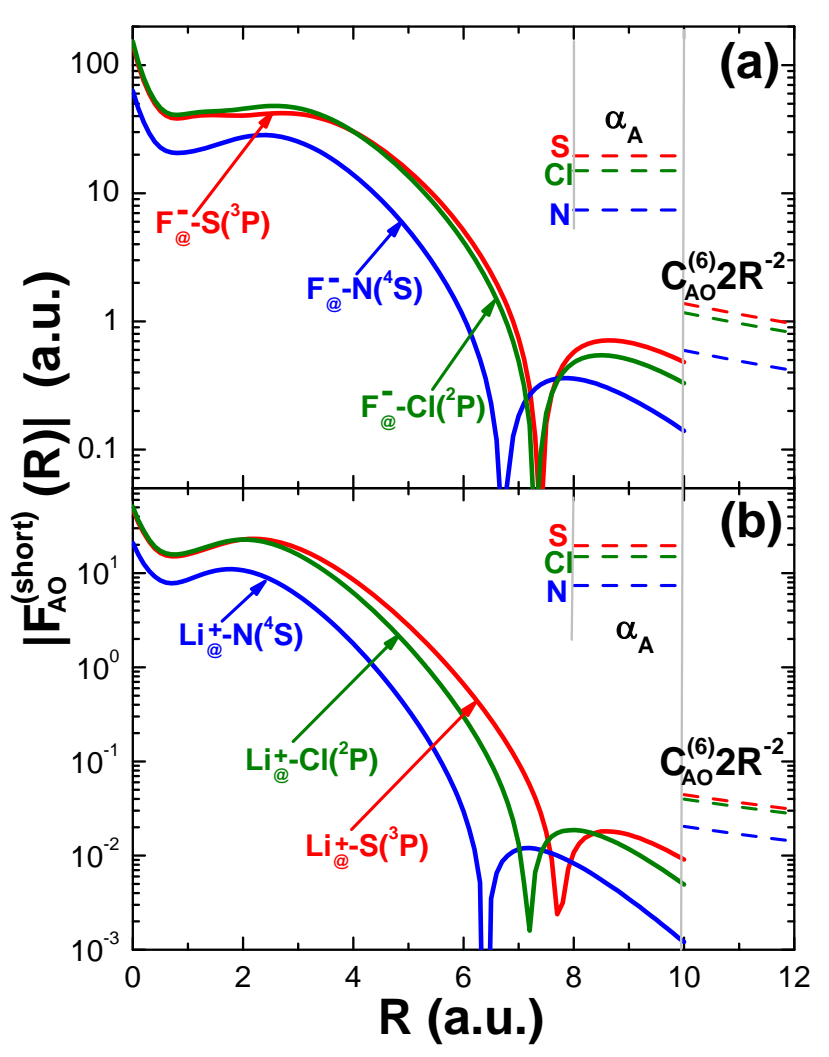

FIG. 3: (Color online) Analogous to Fig. 2 for the binary interaction of open-shell atoms - $\mathrm{N}\left({ }^{4} S\right), \mathrm{S}\left({ }^{3} P\right)$, and $\mathrm{Cl}\left({ }^{2} P\right)$ with: (a) $F_{@}^{-}$anions and (b) $L i_{@}^{+}$cations.

\begin{tabular}{|c|ccccc|}
\hline$A$ & $\alpha_{A}$ & $N_{A}$ & $C_{A-A}^{(6)}$ & $C_{A-F_{\Theta}^{-}}^{(6)}$ & $C_{A-L i^{+}}^{(6)}$ \\
\hline $\mathrm{He}$ & 1.38 & 1.36 & 1.42 & 7.02 & 0.29 \\
$\mathrm{~N}$ & 7.40 & 2.57 & 24.2 & 29.7 & 1.02 \\
$\mathrm{Ne}$ & 2.67 & 3.59 & 6.20 & 14.4 & 0.62 \\
$\mathrm{~S}$ & 19.6 & 4.24 & 134. & 68.9 & 2.22 \\
$\mathrm{Cl}$ & 15.0 & 4.71 & 94.6 & 58.5 & 1.99 \\
$\mathrm{Ar}$ & 11.1 & 5.36 & 64.2 & 48.3 & 1.75 \\
$\mathrm{Kr}$ & 16.7 & 6.45 & 130. & 68.8 & 2.41 \\
$\mathrm{Xe}$ & 27.3 & 5.96 & 261. & 96.2 & 3.11 \\
\hline
\end{tabular}

TABLE III: Dipole polarizability $\alpha_{A}$, number of active electrons $N_{A}$, and $C^{(6)}$ coefficient for the considered atoms $(A)$ [38, 39, 41 and atom-onion pairs. All the values in atomic units. 

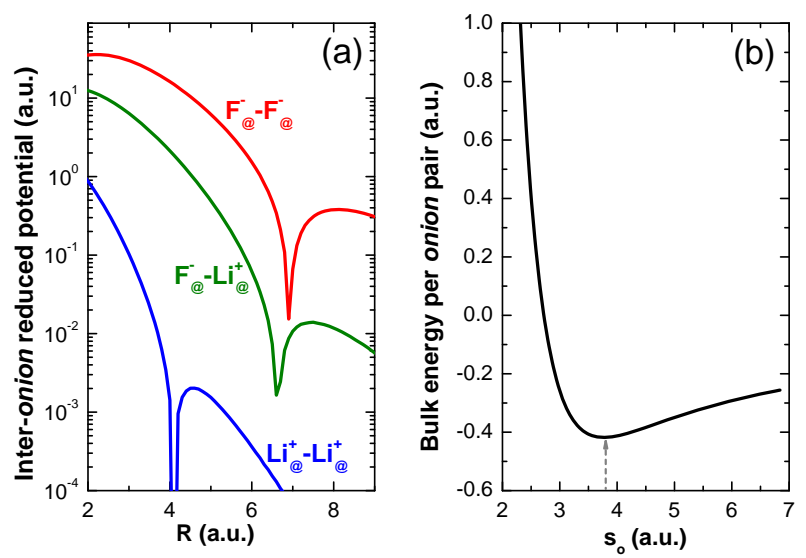

FIG. 4: (Color online) (a) Inter-onion reduced potential (absolute value), as a function of the internuclear distance $R$, for the following onion- pairs: $F_{@}^{-}-F_{@}^{-}, F_{@}^{-}-L i_{@}^{+}, L i_{@}^{+}-L i_{@}^{+}$. Such reduced potentials were derived within the proposed potential model by multiplying by $R\left(1+2 R^{3}\right.$ ) (analogous to Eq. (12)), after extracting the asymptotic Coulomb interaction. (b) Energy per onion- pair at the bulk, as a function of the nearest-neighbor internuclear distance $s_{o}$. The vertical arrow indicates the equilibrium position. 


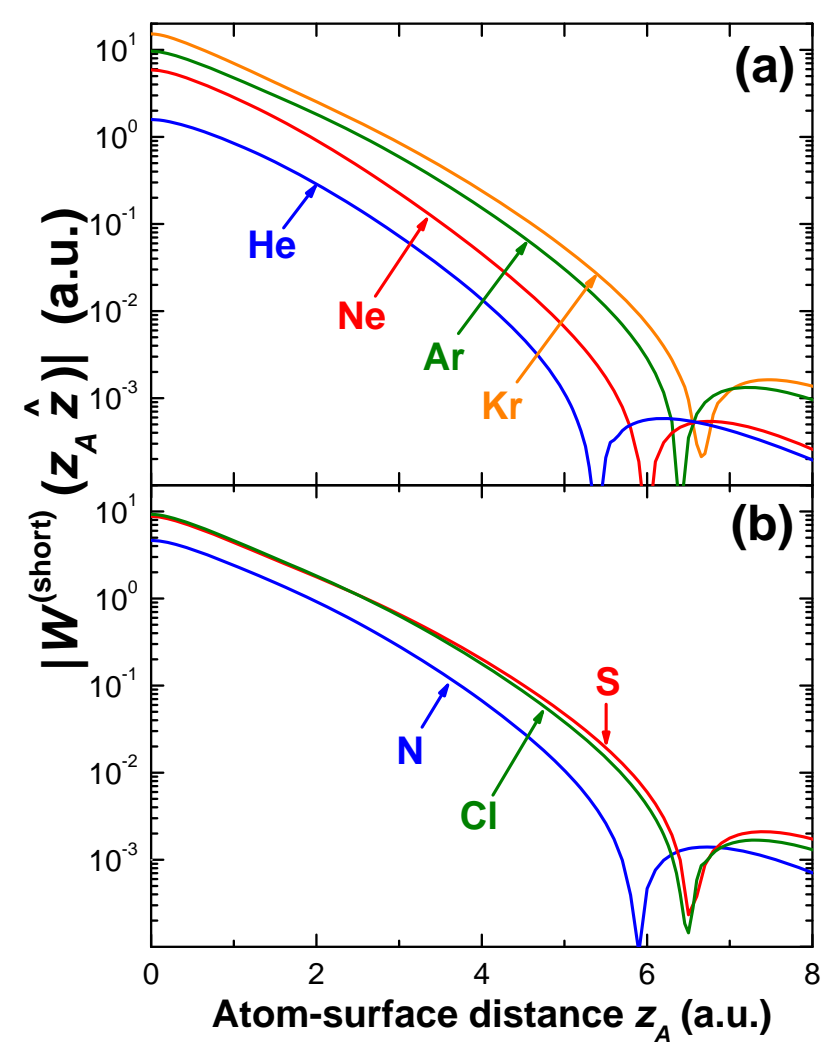

FIG. 5: (Color online) Absolute value of the short-range atom-surface potential $W^{(\text {short })}\left(z_{A} \widehat{\mathbf{z}}\right)$, as defined by Eq. 18, as a function of the atom-surface distance $z_{A}$ measured on top of an $\mathrm{F}^{-}$site. Interactions with different (a) closed-shell and (b) open-shell atoms are displayed with different colors. 


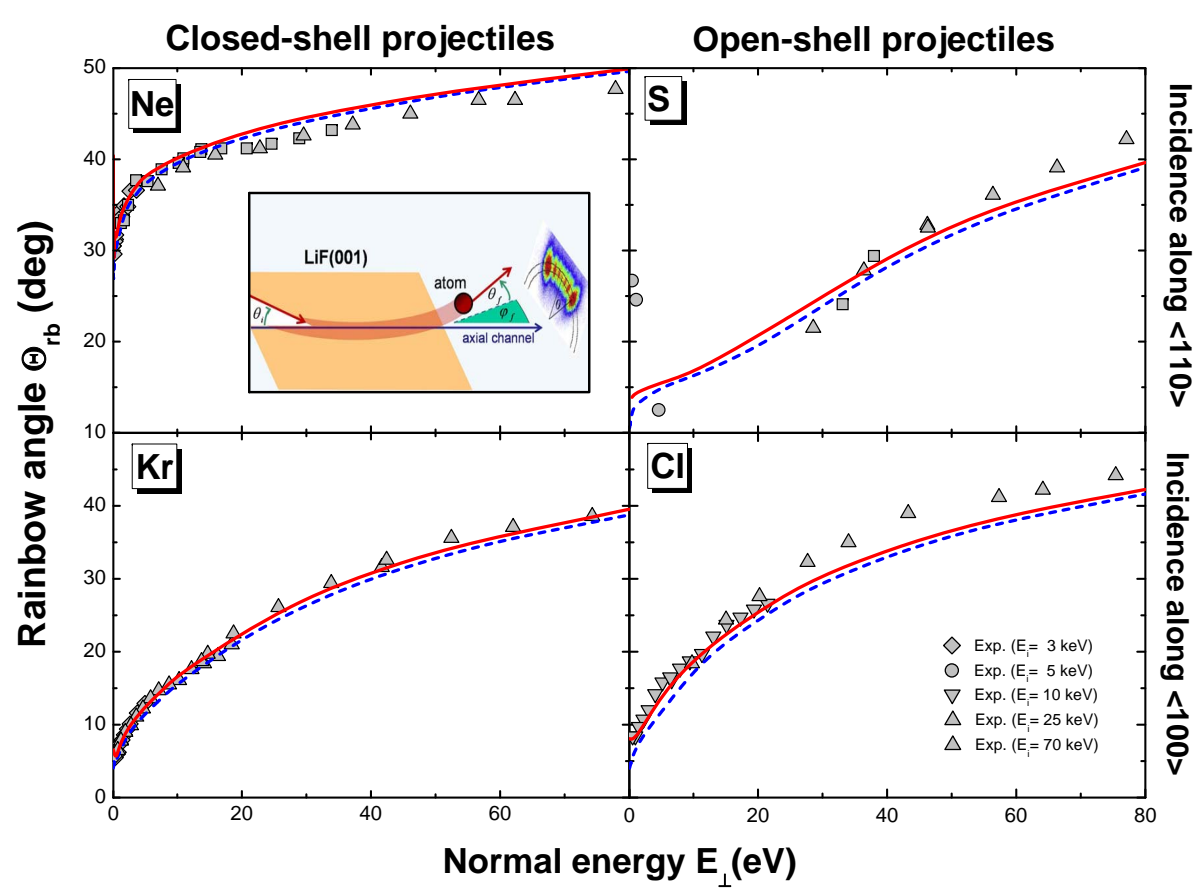

FIG. 6: (Color online) Rainbow deflection angle $\Theta_{\mathrm{rb}}$, as a function of the normal energy $E_{\perp}$, for closed-shell atoms - Ne and $\mathrm{Kr}$ - in the left column, and for open-shell atoms - S and $\mathrm{Cl}$ - in the right column. Panels in the upper and lower rows correspond to the incidence directions $\langle 110\rangle$ and $\langle 100\rangle$, respectively. Red solid line, results obtained from the proposed LLPB3LYP model; blue dashed line, values derived from the LLPB model (neglecting the correlation term); solid symbols, experimental data for different impact energies extracted from Ref. 13, 18. Inset: Depiction of the FAD processes. 


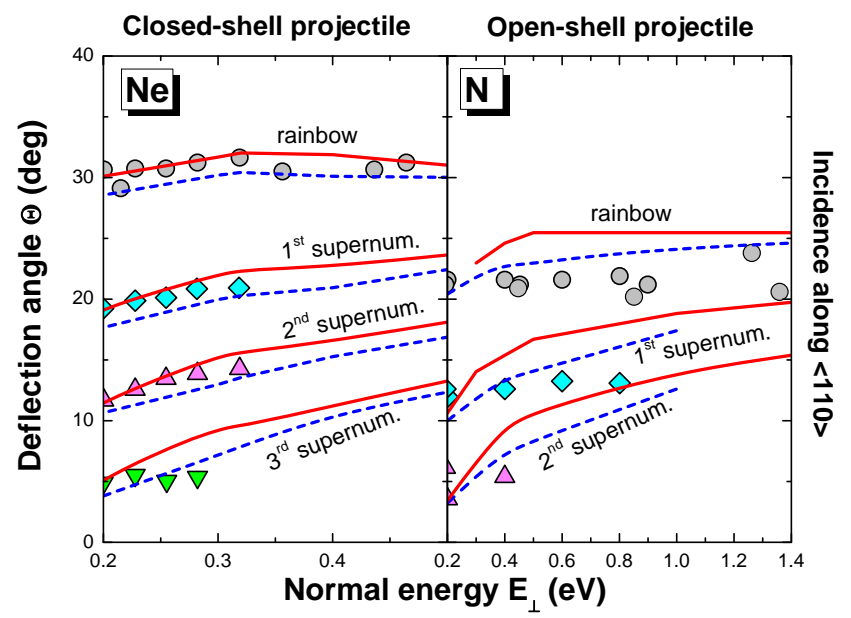

FIG. 7: (Color online) Deflection angles $\Theta$ corresponding to maxima of FAD distributions, as a function of the normal energy $E_{\perp}$, for (a) $\mathrm{Ne}$ (closed-shell) and (b) N (open-shell) atoms scattered along the $\langle 110\rangle$ direction. In both panels, red solid (blue dashed) line, SIVR rainbow and supernumerary rainbow angles derived from the LLPB3LYP (LLPB) models, including (without including) the correlation term. Symbols: experimental data for rainbow (circles) and first (diamonds), second (up triangles), and third (down triangles) supernumerary rainbow angles, extracted from Refs. [13, 48. 